\title{
Avaliação histológica e morfométrica do retalho axial oris angularis e da terapia por ondas de choque aplicados a defeito palpebral experimental em cães
}

\author{
Histologic and morfometric evaluation of oris angularis axial skin flap and extracorporeal \\ shockwave on experimental eyelids defects in dogs
}

\author{
María Guadalupe SERENO ${ }^{1}$; Cláudia Valéria Seullner BRANDÃOํㅜㄹ Bruna Patricia ALMEIDA DA \\ FONSECA $^{1}$; Ana Liz GARCIA ALVES ${ }^{1}$; Giuliana Brasil CROCE ${ }^{1}$; Renée Laufer AMORIM ${ }^{1}$; João \\ Leandro Vera CHIURCIU ${ }^{1}$; Jose Joaquim TITTON RANZANI ${ }^{1}$; Carlos Roberto PADOVANI ${ }^{2}$
}

${ }^{1}$ Faculdade de Medicina Veterinária e Zootecnia da Universidade Estadual Paulista, Botucatu - SP, Brasil

${ }^{2}$ Instituto de Biociências da Universidade Estadual Paulista, Botucatu - SP, Brasil

\begin{abstract}
Resumo
Os retalhos de padrão axial têm como característica significativa vascularização intrínseca, considerada uma vantagem sobre outras técnicas. Considerando que complicações isquêmicas podem afetar os retalhos cutâneos, técnicas de salvamento são descritas, dentre estas, a terapia por ondas de choque extracorpóreas (TOCE), descrita como capaz de modular a vascularização e cicatrização dos retalhos. O presente estudo avaliou histológica e morfometricamente 21 amostras de pele; destas, 14 foram submetidas à confecção do retalho axial, sendo sete tratadas também pela TOCE, obtidas da região distal do retalho axial oris angularis, utilizado para a reconstrução de defeitos palpebrais experimentais extensos em cães. Foram avaliadas também sete amostras de pele normal da mesma região acima descrita (grupo controle). Não foram evidenciadas diferenças histológicas significativas no infiltrado inflamatório e atrofia epidérmica microscopicamente. $\mathrm{Na}$ análise morfométrica, o número de vasos, a área vascular total e a área média foram semelhantes entre os grupos experimentais. $\mathrm{O}$ retalho oris angularis associado ou não à TOCE não apresentou características microscópicas de complicações inflamatórias e atróficas significativas. Sinais de integridade tecidual e vascularização sanguínea adequados foram observados em ambos os grupos tratados, demonstrando efetividade do retalho oris angularis. A aplicação da TOCE no retalho oris angularis, em dose única de $2500 \mathrm{impulsos} \mathrm{a} 0,15 \mathrm{~mJ} / \mathrm{mm}^{2}$ no pós-operatório imediato, não promoveu efeitos colaterais deletérios.
\end{abstract}

Palavras-chave: Avaliação histológica. Morfometria. Retalho oris angularis. Terapia por ondas de choque extracorpóreas. Cães.

\begin{abstract}
Considering that the cutaneous flap can be affected by isquemic complications the extra corporeal shock wave therapy (ESWT) was described as rescue techniques. The present study was developed to analyze histological and with morfometry, twenty one skin samples treated or not with the shock wave therapy, obtained from flap's distal border, used in this study to repair eyelids' experimental defects in dogs. The flap with or without ESWT did not show any histological sign of inflammatory or atrophic alterations. Both group treated showed similar morphometrical characteristics. The ESWT with the protocol used in this study $\left(2500\right.$ impulses at $\left.0,15 \mathrm{~mJ} / \mathrm{mm}^{2}\right)$ did not demonstrate significant clinical outcomes as a rescue technique when applied over the oris angularis flap, however results showed no signals of collateral deleterious effects.
\end{abstract}

Keywords: Histologic evaluation. Morphometry. Oris angularis flap. Extracorporeal shockwave therapy. Dogs.

\section{Introdução}

As pálpebras constituem a primeira barreira de proteção ocular, recobrem o bulbo ocular parcial e externamente, repousam sobre a superfície corneal e conjuntival, removendo corpos estranhos, produzindo de parte do filme lacrimal, distribuindo e drenando o mesmo ${ }^{1}$. Diversos defeitos congênitos, de desenvol-

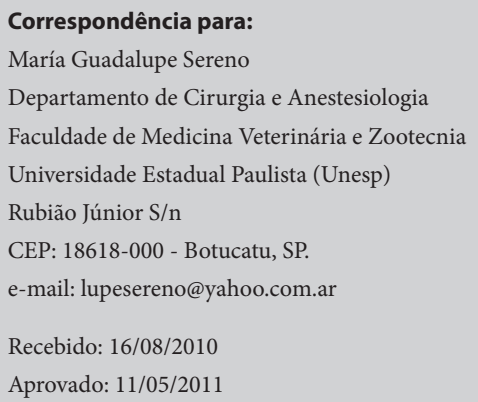


vimento ou adquiridos, podem comprometer a anatomia e funcionalidade das pálpebras, e refletir nas estruturas que as mesmas protegem, podendo afetar a acuidade visual em decorrência da irritação crônica ${ }^{2,3}$.

A reparação de defeitos extensos na face, principalmente os relacionados a neoplasias infiltrativas, constitui um desafio cirúrgico, sendo primordial o conhecimento anatômico da área afetada bem como do tecido adjacente ao defeito para assim preservar a função e a cosmética da região $0^{4,5}$. Muitas vezes retalhos cutâneos são necessários para restaurar defeitos em regiões corporais onde a pele que os circunda não é suficiente. Os retalhos de padrão axial mantêm, no seu pedículo, artérias e veias cutâneas diretas, as quais suprem um território denominado angiossomo, composto pela musculatura cutânea superficial, tecido subcutâneo e pele ${ }^{6}$. A manutenção do pedículo intacto é de vital importância para assegurar que o retalho possa restituir a circulação de áreas isquêmicas ${ }^{7}$.

O retalho auricular caudal ${ }^{8,9}$ e retalho temporal superficial ${ }^{10,11,12}$ foram descritos com resultados satisfatórios na reparação de defeitos da órbita, pálpebras e comissuras palpebrais, associados à excisão de massas tumorais, traumatismos extensos e defeitos que dificilmente fechariam em um único procedimento cirúrgico. Alguns autores estudaram a aplicação clínica do retalho axial oris angularis, com resultados favoráveis para a reconstrução do palato duro ${ }^{13}$ e para a região nasal e facial, nos cães ${ }^{14}$.

Entre as complicações associadas à reconstrução facial mediante retalhos cutâneos, necrose periférica é mencionada com maior frequência na literatura ${ }^{9,15,16}$. A irrigação sanguínea inadequada é a principal causa de necrose $^{7}$; assim, reduzir a isquemia é o foco de várias técnicas de salvamento de retalhos descritas, entre elas a administração exógena de fatores de crescimento ${ }^{17,18}$, oxigenação hiperbárica ${ }^{19}$, e a terapia gênica ${ }^{20,21,22}$.

Nas últimas décadas, a terapia por ondas de choque extracorpóreas (TOCE) tem sido descrita como uma ferramenta útil na modulação da cicatrização que exerce nos diferentes tecidos aos quais é aplicada, ${ }^{23}$ demonstrando interação satisfatória com os tecidos, dose dependência, bom desempenho na sobrevivência de retalhos em modelos experimentais ${ }^{24}$. Acredita-se que a TOCE promove a liberação de fatores de crescimento, assegurando a angiogênese da área comprometida e assim a reparação tecidual ${ }^{25,26}$.

Não há relatos da utilização do retalho de padrão axial oris angularis, para a reconstrução de pálpebras, na literatura veterinária consultada, assim como da aplicação da terapia por ondas de choque extracorpóreas como técnica de salvamento do mesmo na região facial periorbitária.

O presente estudo teve por objetivo avaliar aspectos histológicos e morfométricos da utilização do retalho de padrão axial oris angularis, bem como da aplicação da terapia por ondas de choque extracorpóreas sobre o mesmo, no reparo de defeitos experimentais nas pálpebras inferiores de cães.

\section{Material e Método}

Para a realização do presente estudo o mesmo foi aprovado pela Câmara de Ética em Experimentação Animal (CEEA) da Faculdade de Medicina Veterinária e Zootecnia - UNESP - Campus de Botucatu (protocolo $\mathrm{n}^{\circ}$ 186/2007).

Foram utilizados 14 cães (Canis familiaris - LINNAEUS, 1758), sem raça definida (SRD), de ambos os sexos, adultos jovens (dois a cinco anos), pesando entre oito e $25 \mathrm{~kg}$, sadios, selecionados após exame clínico completo, com especial atenção à superfície ocular e anexos. Foram descartados aqueles que não apresentaram integridade palpebral, bem como irregularidades na pele da face.

Sete cães (14 lados faciais) foram submetidos ao procedimento de reparação de defeitos experimentais nas pálpebras inferiores esquerdas e direitas mediante utilização do retalho de padrão axial oris angularis. Após sorteio, um dos lados faciais foi submetido apenas à realização do retalho (grupo retalho-GR), e o 
outro lado associado à aplicação da terapia por ondas de choque extracorpóreas (grupo TOCE-GT). O grupo controle (GC) foi constituído por sete cães, os quais não foram submetidos a nenhum procedimento.

A medicação pré-anestésica foi realizada com acepromazina $(0,05 \mathrm{mg} / \mathrm{kg})$ e morfina $(0,5 \mathrm{mg} / \mathrm{kg})$; para a indução da anestesia utilizou-se propofol $(5 \mathrm{mg} / \mathrm{kg})$ e isofluorano para manutenção. Após antissepsia da região, utilizou-se um marcador estéril para a delimitação do defeito palpebral experimental, o qual envolveu todo o comprimento da pálpebra inferior e apresentou dois centímetros de largura (Figura 1).

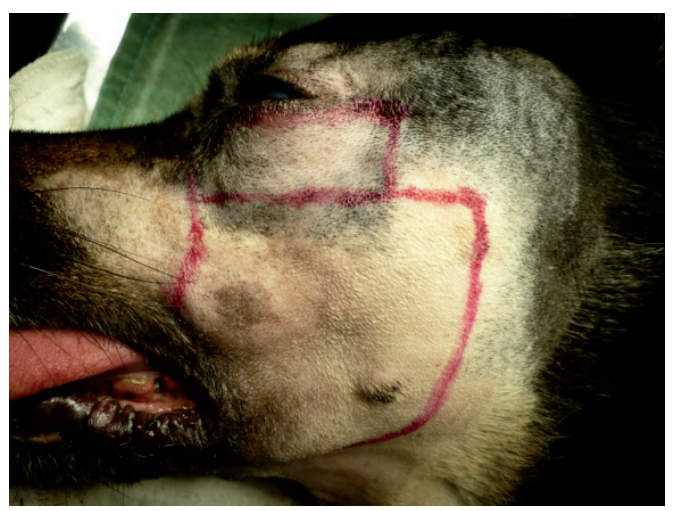

Figura 1 - Imagens fotográficas evidenciando um cão em decúbito lateral direito com defeito palpebral e retalho oris angularis demarcados com tinta estéril

Mantendo-se a base do retalho centrada na comissura bucal, realizaram-se duas incisões paralelas; a ventral, limitada pela borda ventral do ramo mandibular horizontal, e a dorsal, pelo aspecto ventral do arco zigomático; as linhas foram estendidas caudalmente um centímetro após o término do defeito palpebral induzido. Manteve-se a integridade das artérias oris angularis, labial superior e inferior no ângulo da boca, e a dissecção foi realizada subjacente ao músculo platisma cutâneo. O retalho foi deslizado cuidadosamente, e posicionado sobre o defeito cirurgicamente criado. Três pontos subcutâneos foram colocados para facilitar a aproximação das margens cutâneas, as quais foram suturadas com pontos simples separados e náilon 4-0, na borda palpebral e no restante do retalho com náilon 3-0 (Figura 2).

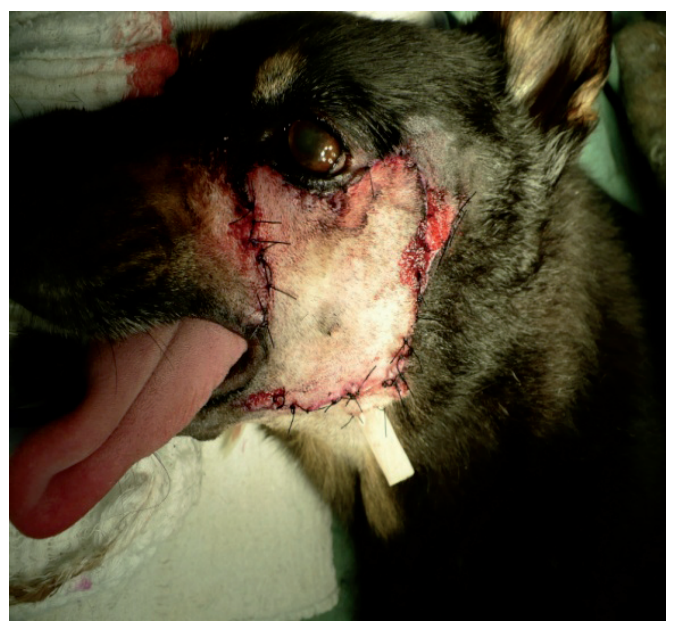

Figura 2 - Aspecto do retalho posicionado e suturado sobre o defeito palpebral

No período pós-operatório imediato, sob anestesia cirúrgica, o GT recebeu uma única aplicação de terapia por ondas de choque (TOCE), sobre a região correspondente à extremidade distal do retalho em relação ao pedículo, sendo considerada a área mais suscetível à necrose.

Aplicaram-se 2500 impulsos a $0,15 \mathrm{~mJ} / \mathrm{mm}^{2}$; a fonte utilizada foi um gerador de ondas de choque eletroidráulico portátil (VersaTron, High Medical Technologies do Brasil Ltda.). O dispositivo de focalização concentrou as ondas a $5 \mathrm{~mm}$ de profundidade desde a superfície de contato; gel de ultrassom foi utilizado para o acoplamento. Um tripé permitiu aperfeiçoar a distância de contato entre o dispositivo de focalização (probe) e a pele dos cães (Figura 3).

Aos dez dias no pós-operatório, todos os animais foram sedados seguindo-se o protocolo pré-anestésico de rotina, para realizar a biopsia de pele. Utilizou-se punch dérmico de $8 \mathrm{~mm}$ de diâmetro. O local padronizado para a obtenção das amostras foi um centímetro lateral e ventral ao canto temporal do olho (Figura 4), área que recebeu a aplicação direta da TOCE nos animais do grupo GT; um ponto simples separado, com fio náilon 3-0, foi aplicado para aproximação da pele. 


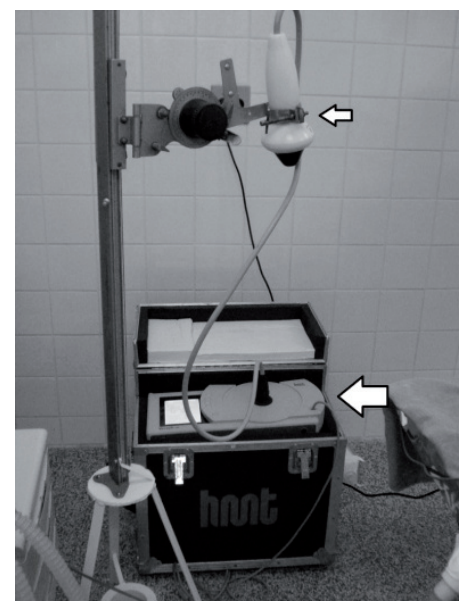

Figura 3 - Gerador de ondas de choque extracorpóreas eletroidráulico portátil (Versatron ${ }^{\circledast}$-HMT) (seta grande) montado sobre um tripé, para otimizar a distância da probe (seta pequena) da pele da face

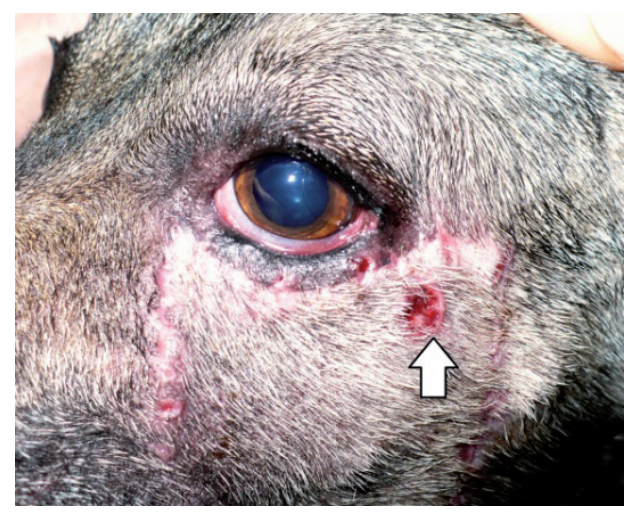

Figura 4 - Local da biopsia de pele no extremo distal do retalho, a 1 $\mathrm{cm}$ lateral do canto temporal do olho (seta)

As amostras de pele foram imersas em solução de formol tamponado a $10 \%$, em frascos separados e identificados durante 24 horas; posteriormente foram retiradas, cortadas sagitalmente em duas peças, colocadas em cassetes plásticos e inseridas em álcool 70\%, até o momento do processamento. Após inclusão em blocos de parafina, as amostras foram cortadas e coradas pela técnica de hematoxilina - eosina (H-E) para posterior avaliação das mesmas.

As avaliações morfológicas e morfométricas foram realizadas em dez campos de cada lâmina, de maneira aleatória, utilizando-se microscópio óptico e aumento de 40x. Os critérios de avaliação morfológica incluí- ram presença de infiltrado inflamatório (mono e polimorfonucleares) e atrofia epidérmica, utilizando-se o sistema de escores para graduar a intensidade das variáveis em 0 : ausente, 1: leve, 2: moderada, 3: intensa.

Os campos de cada lâmina foram capturados fotograficamente e as imagens foram analisadas por meio do programa analisador de imagens Q-win V3 (Leica) versão 2.4 (Microsystems Imaging Solutions Ltd., Cambridge, Inglaterra), para medições manuais e automáticas. $\mathrm{Na}$ análise morfométrica, avaliou-se o número de vasos presentes, a área de cada um deles e a média das áreas dos mesmos. Para todas as variáveis morfológicas e morfométricas foram adotados como valores finais, a média de dez campos analisados em cada lâmina (animal) de cada grupo.

Para as variáveis morfológicas (infiltrado inflamatório e atrofia epidérmica) foi utilizada a técnica de análise de variância não paramétrica de Kruskal-Wallis para o modelo com um fator. As variáveis morfométricas (número de vasos, área de vasos e áreas médias de vasos) foram analisadas pela técnica de análise de variância para o modelo com um fator. Os testes foram realizados a $5 \%$ de significância $(p>0,05)^{27}$.

\section{Resultados e Discussão}

A reparação de defeitos extensos na face constitui um desafio cirúrgico pela pouca disponibilidade de pele existente na região ${ }^{7}$. Alguns retalhos axiais foram descritos para a reconstrução orbitária e maxilofacial em animais domésticos ${ }^{9,10,12}$. O retalho de padrão axial oris angularis foi citado na literatura veterinária para a reconstrução nasal e do palato duro em cães ${ }^{13,14}$. No presente estudo, o retalho oris angularis foi utilizado para a reconstrução de defeitos experimentais realizados nas pálpebras inferiores de cães, abrangendo toda sua extensão e a espessura total da pele das mesmas.

A necrose isquêmica é uma complicação descrita na confecção de retalhos cutâneos, havendo várias técnicas de salvamento dos mesmos, desenvolvidas com a finalidade de evitar tal complicação. A terapia 
por ondas de choque extracorpóreas (TOCE) vem sendo aplicada a vários tecidos com o objetivo de incrementar a perfusão dos mesmos, e assim modular a cicatrizaçãa ${ }^{28}$. Na pele foi relatada sua aplicação em diversas condições, nas quais a irrigação está comprometida ${ }^{24,29}$. No presente estudo a mesma foi aplicada sobre o extremo distal do retalho de padrão axial oris angularis, com a finalidade de aumentar a vascularização da região.

O exame histológico e morfométrico das amostras de pele aos dez dias do pós-operatório, obtidas por biopsia, teve por objetivo identificar possíveis diferenças microscópicas entre os grupos tratados e o controle (Figura 5).

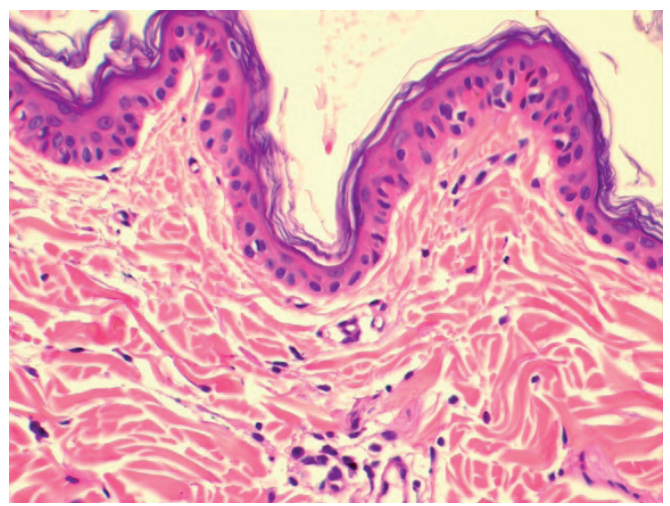

Figura 5 - Corte histológico de pele demonstrando parâmetros normais em um animal do GC (coloração H \& E, obj. 40X)

A presença de células inflamatórias é essencial para dar início ao processo de cicatrização ${ }^{30}$; o infiltrado inflamatório observado no estudo (Figura 6) apresentou um padrão geral leve e sem diferença entre os grupos (Tabela 1), de acordo com os autores que consideram os dez dias após os procedimentos como o declínio da fase inflamatória ${ }^{30}$.

A atrofia epidérmica, caracterizada pelo aspecto delgado da epiderme associado à fina camada de queratina $^{31}$ foi identificada discretamente nos grupos avaliados (Figura 7); esta apresentou grau leve e sem diferença entre os tratamentos (Tabela 2). Segundo
Hargis $^{31}$, em cães é uma característica frequentemente associada a transtornos metabólicos como o hiperadrenocorticismo, portanto, sendo os animais do experimento saudáveis, acredita-se que a mesma representou um achado aleatório não havendo correlação com os tratamentos efetuados.

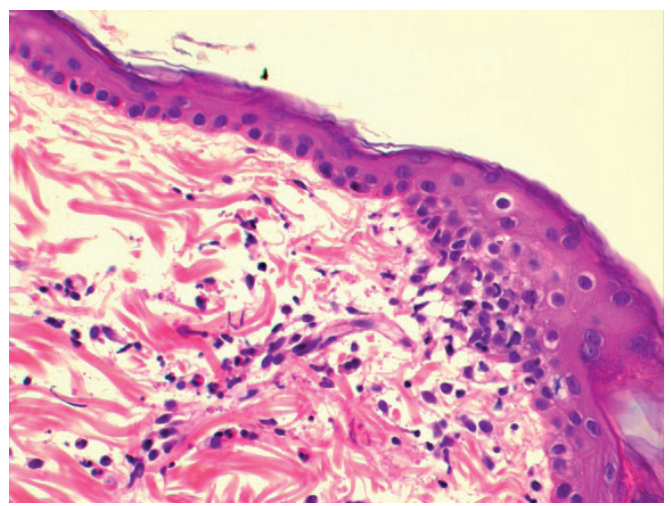

Figura 6 - Corte histológico de pele exemplificando moderado infiltrado inflamatório mononuclear em um animal do GR (coloração H \& E, obj. 40X)

Tabela 1 - Medianas dos escores do infiltrado inflamatório nos diferentes grupos $(\mathrm{G})$ experimentais (GRretalho; GT - TOCE; GC- controle)

\begin{tabular}{cccc}
\hline $\begin{array}{c}\text { Grupos } \\
\text { experimentais }\end{array}$ & GR & GT & GC \\
\hline Escores & 0,5 & 0,7 & 0,3 \\
\hline
\end{tabular}

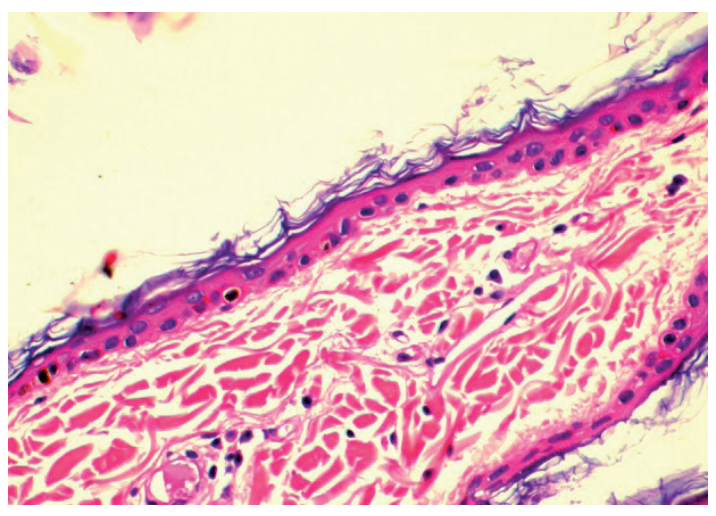

Figura 7 - Corte histológico de pele exemplificando leve atrofia epidérmica em um animal do GT (coloraçã̃o H-E, obj. 40X) 
Tabela 2 - Medianas dos escores da atrofia epidérmica nos diferentes grupos $(\mathrm{G})$ experimentais (GRretalho; GT - TOCE; GC- controle)

\begin{tabular}{cccc}
\hline $\begin{array}{c}\text { Grupos } \\
\text { experimentais }\end{array}$ & GR & GT & GC \\
\hline Escores & 0,2 & 0,1 & 0,1 \\
\hline
\end{tabular}

A presença de vasos sanguíneos, considerando-os como estruturas com luz e presença ou não de hemácias por campo ${ }^{32}$, foram estruturas estabelecidas como medida para avaliar a vascularização das amostras por grupo (Figura 8 A e B). Na análise morfométrica, mediante o programa analisador de imagens Q-win V3 foram contabilizadas as estruturas vasculares segundo Fernandes et al..$^{33}$, e verificou-se um discreto incremento numérico nos grupos tratados (GR e GT) em relação ao GC. Esse resultado pode estar relacionado

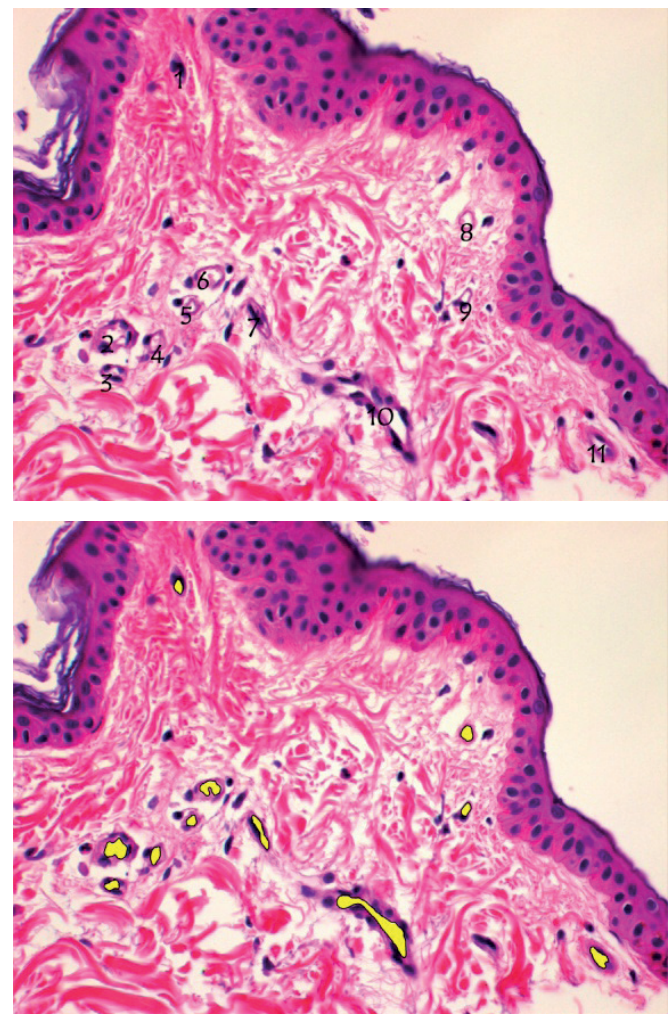

Figura 8 - Corte histológico de pele em um animal do GC ilustrando como foi realizada $(\mathrm{A})$ : contagem de vasos presentes em um campo; (B): medição do lúmen de cada vaso em um campo, obtendo-se a área em $\mu \mathrm{m}^{2}$ (coloração H-E, obj. 40X) como descrito na literatura ${ }^{14}$, ao suprimento sanguíneo adequado observado na confecção do retalho oris angularis decorrente da presença da artéria cutânea direta, bem como angiossomos secundários e terciários no mesmo. Entretanto, diferença significativa não foi observada entre os grupos tratados e o controle, o que demonstra uma aproximação ao padrão normal observada no período de avaliação.

A literatura relata o incremento do número de vasos nas amostras de tecidos submetidos à aplicação da TOCE ${ }^{25,26}$, desigualmente, no presente estudo, não foi possível identificar diferenças significativas entre os grupos tratados, assim como no controle.

A área total do vaso $\left(\mu \mathrm{m}^{2}\right)$ bem como sua área média também foram analisadas pelo programa, obtendo-se as áreas vascularizadas por campo e por amos$\operatorname{tra}^{32}$. Semelhante ao observado com o número de vasos, ou seja, discreto incremento numérico nas áreas vascularizadas, também não se observou diferença estatisticamente significativa entre os grupos (Tabela 3). Huemer et al. ${ }^{29}$ descrevem o aumento da atividade angiogênica após a aplicação da TOCE, observação que não pode ser comprovada diretamente no presente estudo. Acredita-se que tal resultado se deve à boa irrigação do retalho oris angularis ${ }^{14}$.

Tabela 3 - Média e desvio padrão das variáveis: área total, número de vasos e área média do vaso nos diferentes grupos $(\mathrm{G})$ experimentais (GRretalho; GT - TOCE; GC - controle)

\begin{tabular}{cccc}
\hline Variável & GR & GT & GC \\
\hline Área total & $\begin{array}{c}171207,71 \pm \\
52309,63\end{array}$ & $\begin{array}{c}172402,29 \pm \\
41444,58\end{array}$ & $\begin{array}{c}131888,86 \pm \\
38709,87\end{array}$ \\
& & & \\
No de vasos & $7,34 \pm 1,35$ & $6,94 \pm 1,28$ & $6,11 \pm 1,52$ \\
& & & \\
$\begin{array}{c}\text { Área Média } \\
\text { dos vasos }\end{array}$ & $\begin{array}{c}23972,29 \pm \\
6981,58\end{array}$ & $\begin{array}{c}25777,29 \pm \\
5441,67\end{array}$ & $22088,57 \pm$ \\
\hline
\end{tabular}

Com o estudo microscópico dos aspectos inflamatórios, de integridade e estrutura vascular do retalho oris angularis, verificou-se que a TOCE, como técnica 
de salvamento de retalhos, não foi significativa neste estudo, ou seja, não promoveu efeito positivo sobre essas variáveis, à diferença de estudos realizados por Rompe et al. $^{34}$, nos quais infiltrados inflamatórios, proliferação da matriz extracelular e aumento dos capilares foram verificados nos tecidos submetidos à aplicação da TOCE.

Provavelmente devido à boa resposta vascular do retalho axial utilizado, o efeito da TOCE foi minimizado; entretanto, deve-se ressaltar que a sua aplicação na dose estabelecida no presente estudo também não promoveu alterações deletérias ao retalho axial, como

\section{Referências}

1.SAMUELSON, D. A. Ophthalmic anatomy. In: GELATT, K. N. (Ed.). Veterinary ophthalmology. 4. ed. Iowa: Blackwell Publishing, 2007. v. 1, cap. 2, p. 37-148.

2. SLATTER, D. H. Fundamentos de oftalmologia veterinária. 3. ed. São Paulo: Roca, 2005. p. 686.

3. STADES, F. C.; GELATT, K. N. Diseases and surgery of canine eyelid. In GELATT, K. N. (Ed.). Veterinary ophthalmology. 4. ed. Iowa: Blackwell Publishing, 2007. v. 2, cap. 11, p. 563-617.

4. ROBINSON, C. J. Growth factors: therapeutic advances in wound healing. Annals of Medicine, v. 25, p. 535-538, 1993.

5. VAN DER WOERDT, A. Adnexal surgery in dogs and cats. Veterinary Ophthalmology, v. 7, n. 5, p. 284-290, 2004.

6. REMEDIOS, A. Axial pattern flaps, In: FOWLER, D.; WILLIAMS, J. M. (Ed.). Manual of canine and feline wound management and reconstruction. Gloucester: British Small American Veterinary Association, 1999. cap. 8, p. 69-81.

7. PAVLETIC, M. M. Enxertos pediculados. In: SLATTER, D. (Ed.). Manual de cirurgia de pequenos animais. 3. ed. São Paulo: Manole, 2007. v. 1, cap. 23, p. 292-321.

8. SPODNICK, G. J.; HUDSON, L.; CLARK, G. N.; PAVLETIC, M. M. Use of a caudal auricular pattern flap in cats. Journal of the American Veterinary Medical Association, v. 208, n. 10, p. $1679-1682,1996$.

9. STILES, J.; TOWNSEND, W.; WILLIS, M.; MOORE, P. A.; SMITH, E. Use of a caudal auricular axial pattern flap in three cats and one dog following orbital exenteration. Veterinary Ophthalmology, v. 6, n. 2, p. 121-126, 2003.

10.FAHIE, M. A.; SMITH, M. M. Axial pattern flap based on the superficial temporal artery in cats: an experimental study. Veterinary Surgery, v. 26, p. 86-89, 1997.

11.FAHIE, M. A.; SMITH, B. J.; BALLARD, J. B.; MOON, M. L.; SMITH, M. M. Regional peripheral vascular supply based on the superficial temporal artery in dogs and cats. Anatomia Histologia Embryologia, v. 27, p. 205-208, 1998.

12.JACOBI, S.; STANLEY, B. J.; PETERSEN-JONES, S.; DERVISIS, N.; DOMINGUEZ, P. A. Use of an axial pattern flap and nictitans to reconstruct medial eyelids and canthus in a dog. Veterinary Ophthalmology, v. 11, n. 6, p. 395$400,2008$. a intensa lesão inflamatória irreversível descrita por Rompe et al. ${ }^{34}$ para a TOCE a $0,60 \mathrm{~mJ} / \mathrm{mm}^{2}$.

\section{Conclusões}

O retalho oris angularis, associado ou não à TOCE, apresenta sinais de integridade tecidual e vascularização sanguínea adequados, demonstrando efetividade do retalho.

A atuação da TOCE, como técnica de salvamento de retalho, não é significativa no retalho oris angularis, entretanto não promove efeitos colaterais deletérios na aplicação em região facial.

13. BRYANT, K. J.; MOORE, K.; MCANULTY, J. F. Angularis oris axial pattern buccal flap for reconstruction of recurrent fistulae of the palate. Veterinary Surgery, v. 32, p. 113-119, 2003.

14.YATES, G.; LANDON, B.; EDWARDS, G. Investigation and clinical application of a novel axial pattern flap for nasal and facial reconstruction in the dog. Australian Veterinary Journal, v. 85, n. 3, p.113-118, 2007.

15.SMITH, M. M.; PAYNE, J. T.; MOON, M. L.; FREEMAN, L. E. Axial pattern flap based on the caudal auricular artery in dogs. American Journal of Veterinary Research, v. 52, p. 922-925, 1991.

16. TREVOR, P. B.; SMITH, M. M.; WALDRON, D. R.; HEDLUND, C. S. Clinical evaluation of axial pattern skin flaps in dogs and cats: 19 cases (1981-1990). Journal of the American Veterinary Medical Association, v. 201, p. 608-612, 1992.

17.STEED, D. L. The role of growth factors in wound healing. Surgical Clinics of North America, v. 77, p. 575-586, 1997.

18.BANBURY, J.; SIEMIONOW, M.; PORVASNIK, S.; PETRAS, S.; BROWNE, E. Improved perfusion after subcritical ischemia in muscle flaps treated with vascular endothelial growth factor. Plastic Reconstructive Surgery, v. 106, p. 1541-1546, 2000.

19. VIDIGAL, J. Estudo morfológico do músculo sóleo de ratos na isquemia e reperfusão sob oxigenação hiperbárica. 2005. 76 f. Dissertação (Mestrado) - Escola Paulista de Medicina, Universidade Federal de São Paulo, São Paulo, 2005.

20. WALLER, W.; LEE, J.; ZHANG, F.; LINEAWEAVER, W. C. Gene therapy in flap survival. Microsurgery, v. 24, p. 168-173, 2004.

21.ZHANG, F.; WALLER, B. S. W.; LINEAWEAVER, W. C. Growth factors and flap survival. Microsurgery, v. 24, p. 162168,2004

22.GURUNLUOGLU, R.; MEIRER, R.; SHAFIGHI, M.; HUEMER, G.; YILMAZ, B.; PIZA-KATZER H. Gene therapy with adenovirus-mediated VEGF enhances skin flap prefabrication. Microsurgery, v. 25, p. 433-441, 2005.

23. McCLURE, S. R.; DORFMÜLLER, C. Extracorporeal shock wave therapy: theory and equipment. Clinical Techniques in Equine Practice, v. 2, n. 4, p. 348-357, 2003.

24.MEIRER, R.; KAMELGER, F. S.; HUEMER, G. M.; WANNER, S.; PIZA-KATZER, H. Extracorporal shock wave may enhance 
skin flap survival in an animal model. British Journal of Plastic Surgery, v. 58, n. 1, p. 53-57, 2005.

25.WANG, C. J.; YANG, K. D.; CHEN, R. F.; WANG, F. S.; SHEEN-CHEN, S. M. Extracorporeal shockwave promotes growth and differentiation of bone marrow stromal cells towards osteoprogenitors associated with induction of TGFbeta 1 . The Journal of Bone and Joint Surgery, v. 84, p. 457461, 2002.

26. WANG, C. J.; WANG, F. S.; YANG, K. D. Shock wave therapy induces neovascularization at the tendon-bone junction. A study in rabbits. Journal of Orthopaedic Research, v. 21, p. 984-989, 2003.

27.ZAR, J. H. Biostatistical analysis. 4. ed. New Jersey: PrenticeHall, 1999. 663 p.

28.SCHADEN, W.; THIELE, R.; KÖLPL, C.; PUSCH, A. ESWT in skin lesions. Newsletter of Extracorporeal Shockwave Therapy, v. 2, p. 13-15, 2006.

29.HUEMER, G. M.; MEIRER, R.; GURUNLUOGLU, R.; KAMELGER, F. S.; DUNST, K. M.; WANNER, S.; PIZAKATZER, H. Comparison of the effectiveness of gene therapy with transforming growth factor-b or extracorporal shock wave therapy to reduce ischemic necrosis in an epigastric skin flap model in rats. Wound Repair and Regeneration, v. 13, n. 3, p. 262-268, 2005.

30. WITTE, M. B.; BARBUL, A. General principles of wound healing. Surgical Clinics of North America, v. 77, n. 3, p. 509-528, 1997.

31.HARGIS, A. M. Sistema tegumentar. In: CARLTON, W. W.; MCGAVIN, M. D. (Ed.). Patologia veterinária especial de Thomson. 2. ed. São Paulo: Manole, 1995. cap. 11, p. 486-541.

32.FERREIRA, M. A. N. D. Avaliação da angiogênese, inflamação e crescimento tumoral em camundongos com deleção gênica do PAF (PAFR-KO). 2006. Tese (Doutorado) - Instituto de Biociências, Universidade Federal de Minas Gerais, Belo Horizonte, 2006.

33. FERNANDES, J. B. V. D.; MATAYOSHI, S.; OSAKA, J. T.; TOLOSA, E. C. M.; NUNES, T. P.; MOURA, E. M. Análise comparativa entre esclera tratada com glicerina e esclera tratada com irradiação gama, álcali e glicerina em reconstrução de cavidade anoftálmica: estudo experimental em coelhos. Arquivos Brasileiros de Oftalmologia, v. 70, n. 4, p. 639-647, 2007.

34.ROMPE, J. D.; KIRKPATRICK, C. J.; KUELLMER, K.; SCHWITALLE, M.; KRISCHEK, O. Dose-related effects of shock waves on rabbit tendo achillis. Journal of Bone and Joint Surgery, v. 80, p. 546-552, 1998. 